VoL. 41 (1990) [381-386]

\title{
NORMS IN POLYNOMIAL RINGS
}

\author{
G. MYERSON
}

We give a formula for the norm on a polynomial ring modulo an ideal in terms of the zero-set of the ideal. We hint at the relation to resultants.

\section{DEFINITIONS AND STATEMENT OF THEOREM}

Let $A$ be a ring (by which we mean a commutative ring with unity). Let $B$ be a ring containing $A$, and suppose that, as an $A$-module, $B$ is finitely-generated and free. Let $b$ be any element of $B$; then multiplication by $b$ is an $A$-linear operator $T_{b}$ on $B$. The norm from $B$ to $A$ of $b$, written $N_{A}^{B} b$, is defined to be the determinant of $T_{b}$.

Perhaps the most familiar example is that in which $A$ is the rationals and $B$ is a number field; $N_{A}^{B} b$ then coincides with the field norm of algebraic number theory.

In what follows, we write $A_{n}$ for $A\left[x_{1}, \ldots, x_{n}\right]$.

Theorem. Let $A$ be an integral domain, and let $I$ be an ideal in $A_{n}$ such that $B=A_{n} / I$ is, as an $A$-module, finitely-generated and free. Let $k$ be an algebraically closed field containing $A$, and let $Z(I)$ be the set of all zeros of $I$ over $k$. Then $Z(I)$ is finite and, if $f$ is in $A_{n}$, then

$$
N_{A}^{B} \bar{f}=\prod_{P \in Z(I)} f(P)^{m_{P}}
$$

where $\bar{f}=f+I$ is the image of $f$ in $B$, and $m_{P}$ is the multiplicity of $P$ as a zero of $I$.

Multiplicity is used here in the standard sense of algebraic geometry - we elaborate on this in the course of the proof. We note that the condition on $I$ is quite restrictive; for example, if $A$ is the ring of integers and $n$ is 1 then $I$ must be principal with monic generator. Steve Schanuel has suggested that $B$ need only be projective, not free, but we have not explored this idea.

Received 6 June 1989

Copyright Clearance Centre, Inc. Serial-fee code: 0004-9729/80 \$A2.00+0.00. 


\section{Proof of part of the theorem}

We believe that the finiteness of $Z(I)$ (under the hypotheses of the theorem) is due to Gröbner [3]. For the reader's convenience, we present a simple proof.

Proof: (of the finiteness of $Z(I)$ ). For a given $j, 1 \leqslant j \leqslant n$, we consider the elements $1, x_{j}, x_{j}^{2}, \ldots$, of $A_{n}$. Their images $1, \bar{x}_{j}, \bar{x}_{j}^{2}, \ldots$ in $B$ cannot be $A$-linearly independent, since $B$ is finitely-generated as an $A$-module; thus there exists a positive integer $r$ and elements $a_{0}, \ldots, a_{r}$ of $A$ such that $a_{0}+a_{1} \bar{x}_{j}+\ldots+a_{r} \bar{x}_{j}^{r}=0$ in $B$. Let $f_{j}(\underline{x})=a_{0}+a_{1} x_{j}+\ldots+a_{r} x_{j}^{r}$; then $\bar{f}_{j}=0$, so $f_{j} \in I$. Now let $P=\left(\alpha_{1}, \ldots, \alpha_{n}\right)$ be in $Z(I)$. Then $f_{j}(P)=0$, so $a_{0}+a_{1} \alpha_{j}+\ldots+a_{r} \alpha_{j}^{r}=0$, so there are only finitely many possible values for $\alpha_{j}$. But $j$ was arbitrary, so there are only finitely many points in $Z(I)$.

\section{Change of base, and Nullstellensatz}

We wish to reduce the theorem to the case where $A=k$, that is, where $A$ is an algebraically closed field.

Lemma 1. Let $A$ be a ring, let $I$ be an ideal in $A_{n}$, let $B=A_{n} / I$. Let $A^{\prime}$ be a ring containing $A$, with $A^{\prime} \cap A_{n}=A$. Let $I^{\prime}$ be the ideal generated by $I$ in $A_{n}^{\prime}=A^{\prime}\left[x_{1}, \ldots, x_{n}\right]$, let $B^{\prime}=A_{n}^{\prime} / I^{\prime}$. Then

(1) $B^{\prime} \simeq B \otimes_{A} A^{\prime}$,

(2) if $B$ is, as an $A$-module, finitely-generated and free with basis $\left\{f_{1}+\right.$ $\left.I, \ldots, f_{r}+I\right\}$ then $B^{\prime}$ is, as an $A^{\prime}$-module, finitely-generated and free with basis $\left\{f_{1}+I^{\prime}, \ldots, f_{r}+I^{\prime}\right\}$, and, in this case,

(3) if $g$ is in $A_{n}$, then $N_{A^{\prime}}^{B^{\prime}}\left(g+I^{\prime}\right)=N_{A}^{B}(g+I)$.

PROOF: (with our thanks to Jonathan Hillman). Tensor the exact sequence $0 \longrightarrow I \longrightarrow A_{n} \longrightarrow B \longrightarrow 0$ over $A$ with $A^{\prime}$ to obtain the top row in the following commutative diagram:

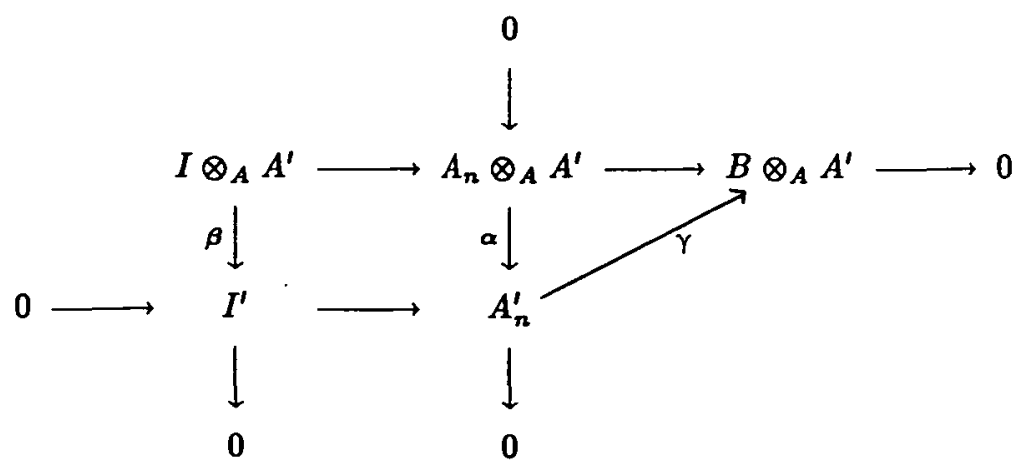


The map $\alpha$ is defined by $\alpha\left(f \otimes a^{\prime}\right)=a^{\prime} f$ and linearity; it is the canonical identification of $A_{n} \otimes_{A} A^{\prime}$ with $A_{n}^{\prime}$. The map $\beta$ is defined by $\beta\left(i \otimes a^{\prime}\right)=a^{\prime} i$ and linearity; it is surjective since every element of $I^{\prime}$ is a sum of terms of the form $a^{\prime} i$ with $a^{\prime} \in A^{\prime}$ and $i \in I$. The map $\gamma$ is defined to make the triangle commute.

A routine diagram chase establishes that $0 \longrightarrow I^{\prime} \longrightarrow A_{n}^{\prime} \longrightarrow B \otimes_{A} A^{\prime} \longrightarrow 0$ is exact, whence $B \otimes_{A} A^{\prime} \simeq A_{n}^{\prime} / I^{\prime}=B^{\prime}$. The rest of the lemma follows from basic facts about tensor products amd the definition of the norm.

It follows from Lemma 1 that in proving the theorem we may assume $A=k$ is an algebraically closed field. We shall have need of the Hilbert Nullstellensatz, which we state as it appears in [5].

LEMMA 2. If $J$ is an ideal of $k_{n}=k\left[x_{1}, \ldots, x_{n}\right]$, if $f \in k_{n}$, and if $Z(J) \subseteq Z(f)$ then there is a non-negative integer $m$ such that $f^{m}$ is in $J$.

\section{Proof of The theorem By CoMmUtative algebra}

We take as given the hypotheses of the theorem, with $A=k$.

LEMMA 3. I has a reduced primary decomposition, $I=\bigcap_{j} Q_{j}$.

Proof: $k_{n}$ is Noetherian.

LEMma 4. For each $j, Z\left(Q_{j}\right)$ is a single point.

PROOF: $Z\left(Q_{j}\right)$ is certainly a finite set, since $Z(I)=\bigcup_{j} Z\left(Q_{j}\right)$. Suppose $Z\left(Q_{j}\right)=$ $S \cup T$, where $S$ and $T$ are disjoint and non-empty. Construct $f, g$ in $k_{n}$ such that $f$ vanishes on $S$ but not on $T$, and $g$ vanishes on $T$ but not on $S$ (such $f$ and $g$ exist since $S$ and $T$ are finite sets and $k$ is an infinite field). Then $Z\left(Q_{j}\right) \subseteq Z(f g)$, so, by the Nullstellensatz, $(f g)^{m}$ is in $Q_{j}$ for some non-negative integer $m$. Since $Q_{j}$ is primary, some power of $f$ or $g$ is in $Q_{j}$; but this is absurd, since $f$ does not vanish on $T$ and $g$ does not vanish on $S$.

LEMMA 5. The $Q_{j}$ are pairwise relatively prime.

Proof: For each $j$, let $Z\left(Q_{j}\right)=\left\{P_{j}\right\}$. If $r \neq s$ then $P_{r} \neq P_{s}$, since $I=\bigcap_{j} Q_{j}$ is a reduced primary decomposition. Assume $P_{r}$ and $P_{0}$ differ in coordinate $\ell$, that is, $P_{r}=\left(\alpha_{1}, \ldots, \alpha_{n}\right), P_{\ell}=\left(\beta_{1}, \ldots, \beta_{n}\right)$, with $\alpha_{\ell} \neq \beta_{\ell}$. Let $f(\underline{x})=x_{\ell}-\alpha_{\ell}$, let $g(\underline{x})=x_{\ell}-\beta_{\ell}$. Then $f\left(P_{r}\right)=0$, so by the Nullstellensatz $f^{u}$ is in $Q_{r}$ for some nonnegative integer $u$; similarly, $g^{v}$ is in $Q$, for some non-negative integer $v$. It follows that

$$
0 \neq\left(\alpha_{\ell}-\beta_{\ell}\right)^{u+v-1}=(g-f)^{u+v-1}=f^{u} F+g^{v} G
$$

for some $F, G$ in $k_{n}$. Thus $Q_{r}+Q_{\imath}=k_{n}$. 
LEMMA 6. $B \simeq \bigoplus_{j} k_{n} / Q_{j}$ (isomorphism as $k$-algebras).

Proof: Chinese Remainder Theorem.

Now let $B_{j}=k_{n} / Q_{j}$, and let $m_{j}$ be the dimension of $B_{j}$ as a $k$-vector space this is the standard definition of the multiplicity of $P$ as a zero of $I$.

Proof of The Theorem: Let $f \in k_{n}$. Then for each $j,\left(f+Q_{j}\right) B_{j} \subseteq B_{j}$, so $N_{k}^{B} \bar{f}=\prod_{j} N_{k}^{B_{j}}\left(f+Q_{j}\right)$. Let $T_{j}$ be the restriction to $B_{j}$ of the linear operator, "multiplication by $\bar{f}$ ", and let $\lambda$ be an eigenvalue of $T_{j}$ with corresponding eignevector $b \neq 0$. Thus $\left(f+Q_{j}\right) b=\lambda b$. Let $b=v+Q_{j}$ for some $v \in k_{n}$; then $(f-\lambda) v \in Q_{j}$. Now $b \neq 0$ implies $v \notin Q_{j}$. Since $Q_{j}$ is primary, there is a positive integer $m$ such that $(f-\lambda)^{m} \in Q_{j}$. Thus $\left(f\left(P_{j}\right)-\lambda\right)^{m}=0$, so $\lambda=f\left(P_{j}\right)$. Hence $N_{k}^{B_{j}}\left(f+Q_{j}\right)=$ $f\left(P_{j}\right)^{m_{j}}$, whence $N_{k}^{B} \bar{f}=\prod_{j} f\left(P_{j}\right)^{m_{j}}$.

\section{PROOF OF THE THEOREM BY LINEAR ALGEBRA}

We present a second proof which does not involve primary ideals or the Chinese Remainder Theorem (at least, not overtly). We let $Z(I)=\left\{P_{1}, \ldots, P_{\ell}\right\}$.

Lemma 7. Given $f, g$ in $k_{n}$ with $f g \in I$, if $Z(f) \cap Z(I)=\phi$, then $g \in I$.

Proof: Let $h=\prod_{j=1}^{\ell}\left(f-f\left(P_{j}\right)\right)$. Then $Z(I) \subseteq Z(h)$ so, by the Nullstellensatz, $h^{m}$ is in $I$ for some non-negative integer $m$. Thus $h^{m} g$ is in $I$. Now $h^{m}=f r+c$ for some $r \in k_{n}$ and some non-zero $c$ in $k-$ in fact $c=(-1)^{\ell m}\left[\prod_{j} f\left(P_{j}\right)\right]^{m}$. So from $h^{m} g$ in $I$ we deduce $f r g+c g$ in $I$, whence $c g$ is in $I$, whence $g$ is in $I$.

LеммA 8. Let $T_{f}$ be the linear operator on $B$ given by multiplication by $\bar{f}$. Then the eignevalues of $T_{f}$ are precisely the quantities $f\left(P_{j}\right), j=1,2, \ldots, \ell$.

Proof: Assume $T_{f} b=\lambda b$ for some non-zero $b$ in $B$ and some $\lambda$ in $k$. Choose $g$ in $k_{n}$ such that $b=g+I$; note that $b \neq 0$ implies $g$ is not in $I$. Then $(f-\lambda) g$ is in $I$. By Lemma 7, $Z(f-\lambda) \cap Z(I) \neq \emptyset$; hence, $\lambda=f\left(P_{j}\right)$ for some $j$.

Conversely, for each $j$, choose $u_{j}$ in $k_{n}$ such that $u_{j}\left(P_{r}\right)=\delta_{j r}$. Such polynomials are easily constructed explicitly, and we omit the details. Let $v_{j}=\left(f-f\left(P_{j}\right)\right) u_{j}$. Then $Z(I) \subseteq Z\left(v_{j}\right)$, so, by the Nullstellensatz, $v_{j}^{m}=\left(f-f\left(P_{j}\right)\right)^{m} u_{j}^{m}$ is in $I$ for some positive integer $m$. On the other hand, $u_{j}^{m}$ is not in $I$, since $u_{j}^{m}\left(P_{j}\right) \neq 0$. So there is an integer $r, 0 \leqslant r<m$, such that $\left(f-f\left(P_{j}\right)\right)^{r} u_{j}^{m}$ is not in $I$ but $\left(f-f\left(P_{j}\right)\right)^{r+1} u_{j}^{m}$ is. Let $w_{j}=\left(f-f\left(P_{j}\right)\right)^{r} u_{j}^{m}$; then $\bar{w}_{j}$ is an eigenvector for $T_{f}$ with corresponding eigenvalue $f\left(P_{j}\right)$. For, $T_{f} \bar{w}_{j}=f w_{j}+I=\left(f-f\left(P_{j}\right)\right) w_{j}+f\left(P_{j}\right) w_{j}+I=f\left(P_{j}\right) w_{j}+I=$ $f\left(P_{j}\right) \bar{w}_{j}$. 
It follows from Lemma 8 that for every $f$ in $k_{n}$ there exist positive integers $m_{1}, \ldots, m_{l}$ such that $N_{k}^{B} \bar{f}=\prod f\left(P_{j}\right)^{m_{j}}$. To conclude the proof of the theorem it remains only to show that the $m_{j}$ can be chosen independently of $f$.

ProOF OF THE THEOREM: Choose $h$ in $k_{n}$ such that $r \neq s$ implies $h\left(P_{r}\right) \neq h\left(P_{s}\right)$. Let $B=B_{1} \oplus \ldots \oplus B_{\ell}$, where $B_{j}$ is the eigenspace of $T_{h}$ corresponding to the eigenvalue $h\left(P_{j}\right), j=1, \ldots, \ell$. Let $m_{j}=\operatorname{dim}_{k} B_{j}$. By Lemma 8 , each $m_{j}$ is positive. It is clear that $N_{k}^{B} \bar{h}=\prod h\left(P_{j}\right)^{m_{j}}$.

Now for $j=1, \ldots, \ell$ let $u_{j}$ be as in the proof of Lemma 8. By the argument of that lemma, $\left(h-h\left(P_{j}\right)\right)^{m} u_{j}^{m}$ is in $I$ for some integer $m$. Equivalently, $\left(T_{h}-h\left(P_{j}\right)\right)^{m} \bar{u}_{j}^{m}=$ 0 , so $\bar{u}_{j}^{m}$ is a non-zero element of $B_{j}$.

Now let $f$ be an arbitrary element of $k_{n}$. Since $T_{h}$ and $T_{f}$ commute, $B_{j}$ is an eigenspace for $T_{f}$. Let $\lambda$ be the corresponding eigenvalue. Then there is an integer $r$ such that $\left(T_{f}-\lambda\right)^{r} \bar{u}_{j}^{m}=0$, that is $(f-\lambda)^{r} u_{j}^{m}$ is in $I$. Evaluating at $P_{j}$, and recalling that $u_{j}\left(P_{j}\right) \neq 0$, we see $\left(f\left(P_{j}\right)-\lambda\right)^{r}=0$, so $\lambda=f\left(P_{j}\right)$. The theorem now follows. [

We note that this proof presents an alternative method of viewing the multiplicity of a zero $P_{j}$ of the ideal $I$, namely, as the dimension of the generalised eigenspace $B_{j}$ corresponding to the eigenvalue $h\left(P_{j}\right)$ of an operator $T_{h}$, where $h$ is such that $r \neq s$ implies $h\left(P_{r}\right) \neq h\left(P_{s}\right)$.

\section{REsUltaNts}

Let $A$ be a commutative ring with unity. Let $f$ and $g$ be polynomials with coefficients in $A$. The resultant of $f$ and $g$, written $R(f, g)$, is defined to be the determinant of the Sylvester matrix; this is the matrix

$$
\left[\begin{array}{cccccccc}
a_{n} & a_{n-1} & & \ldots & a_{0} & & & \\
& a_{n} & & \ldots & & a_{0} & & 0 \\
0 & & \ddots & & & & \ddots & \\
& & & a_{n} & \ldots & & & a_{0} \\
b_{m} & b_{m-1} & & \ldots & b_{0} & & & \\
& b_{m} & & \ldots & & b_{0} & & 0 \\
0 & & \ddots & & & & \ddots & \\
& & & b_{m} & & & & b_{0}
\end{array}\right]
$$

where $f(x)=\sum_{j=0}^{n} a_{j} x^{j}$ and $g(X)=\sum_{j=0}^{m} b_{j} x^{j}, a_{n} \neq 0, b_{m} \neq 0$ (in the matrix the coefficients of $f$ fill $m$ rows, and the coefficients of $g$ fill $n$ rows).

If $A$ is an integral domain then there are well-known expressions for $R(f, g)$ in terms of the zeros of $f$ and/or $g$, for example

$$
R(f, g)=a_{n}^{m} \prod g(\alpha),
$$


where $\alpha$ runs through the zeros of $f$ in a splitting field containing $A$, with multiplicities. Comparing this with the theorem yields

Corollary 1. Let $A$ be an integral domain. Let $f$ in $A_{1}$ be monic. Let $B=A_{1} /(f)$. Then for all $g$ in $A_{1}$ we have

$$
R(f, g)=N_{A}^{B} \bar{g} .
$$

Both sides of (2) are defined in terms of the coefficients of $f$ and $g$ alone, from which it follows that (2) holds under the weaker hypothesis that $A$ be a commutative ring with unity. This attractive result has been discovered independently several times. Professor Schinzel informs me that a formula equivalent to (2) appears in a work of Cebotarev [2] to which I have not had access; since then it has appeared in $[6,4,9,1$, 10], and, we regret, [7].

We would like to generalise Cebotarev's result to multivariate polynomial rings. There are difficulties with resultants of systems of multivariate polynomials that do not arise in the one-variable case, but our theorem suggests that here, too, norms and resultants are very closely related - see also the expression for the resultant given by Netto [8]. We hope in a later paper to expand on the relation between the norm as presented here and the resultant of a system of multivariate polynomials.

\section{REFERENCES}

[1] S. Barnett, 'Greatest common divisor of two polynomials', Linear Algebra Appl. 3 (1970), 7-9.

[2] N.G. Cebotarev, Teorija Galua (Mathematika w Monografijach, Serija Obsorow I, Moskwa, Leningrad, 1936).

[3] W. Gröbner, Moderne algebraische Geometrie (Springer, Vienna and Innsbruck, 1949).

[4] R.E. Kalman, 'Mathematical description of linear dynamical systems', SIAM J. Control 1 (1963), 152-192.

[5] S. Lang, Algebra (Addison Wesley, Reading, Mass., 1965).

[6] N.H. McCoy, 'Divisors of zero in matric rings', Bull.Amer.Math.Soc. 47 (1941), 166-172.

[7] G. Myerson, 'On resultants', Proc. Amer. Math. Soc. 89 (1983), 419-420.

[8] E. Netto, Vorlesungen über Algebra, vol II (Leipzig, 1900).

[9] H. Schmidt, 'Bemerkung zur elementaren Algebra : I. Restklassenring und Resultante', Bayer. Akad. Wiss. Math. - Natur. Kl. Sitzungsber. 1966 II (1967), 167-172.

[10] W.G. Vogt and N.K. Bose, 'A method to determine whether two polynomials are relatively prime', IEEE Trans. Automat. Control AC-15 (1970), 379-380. 